\title{
TARIFAS E QUOTAS: UMA ANÁLISE COM TEORIA DOS JOGOS*
}

\author{
André Luís Rossi de Oliveira**
}

\section{Resumo}

Este trabalho procura demonstrar que o resultado tradicional de equivalência entre tarifas e quotas, encontrado na teoria do comércio internacional, não se verifica quando passamos ao contexto de jogos finitamente repetidos com utilidades médias.

Nesse sentido, mostramos que o jogo de tarifas com utilidades médias apresenta equilíbrios perfeitos em subjogos sobre a fronteira de possibilidades de utilidades, enquanto que o único equilíbrio perfeito do jogo de quotas é a repetição do equilíbrio de Nash de um só período.

Isto nos leva à conclusão de que, utilizando qualquer uma das definiçōes de equivalência disponíveis, não há equivalência entre tarifas e quotas quando pensamos no comércio internacional como um jogo finitamente repetido com utilidades médias. Este resultado já existe na literatura para jogos com utilidades descontadas.

\section{Abstract}

This paper tries to demonstrate that the traditional result of the equivalence of tariffs and quotas, as described in the theory of international trade, is no longer valid when we are in the context of finitely repeated games with average payoffs.

In this way, we show that the tariffs game with average payoffs has subgame perfect equilibria on the payoffs possibilities frontier, while the only subgame perfect equilibrium of the quotas game is the repetition of the one shot Nash equilibrium.

\footnotetext{
*Este artigo foi baseado na minha dissertação de mestrado, que tem o mesmo título, defendida em 01/07/91 na Universidade de Brasília. Gostaria de agradecer ao professor Neantro Saavedra Rivano, meu orientador, que me sugeriu perseguir o tema de equivalência entre tarifas e quotas, pelas inúmeras contribuiçōes a este artigo e à minha tese. Também quero agradecer aos professores Stephen de Castro, Mirta Bugarin e Jean-Luc Rosinger, membros da banca examinadora da minha dissertação, pelos valiosos comentários que permitiram o aprimoramento deste trabalho. Por fim, agradeço a dois pareceristas anônimos pelas críticas esclarecedoras, que possibilitaram a correção de várias falhas. As remanescentes são de minha exclusiva responsabilidade.

**Mestre em Economia pela Universidade de Brasília e aluno do curso de $\mathrm{PhD}$ em Economia da Universidade de Illinois em Urbana-Champaign.
} 
This leads us to the conclusion that, using whatever available definition, there is no equivalence of tarifes and quotas whem we think about the international trade as a finitelly repeated game with average payoffs. This result already exists in the literature for games with discounted payoffs.

\section{Introdução.}

Um dos problemas mais atuais concernentes ao comércio mundial é o das políticas protecionistas. Vive-se um momento em que a pressão por políticas liberais de comércio convive com a resistência de países desenvolvidos, como o Japão e os membros da Comunidade Econômica Européia, em flexibilizar suas políticas comerciais.

$O$ intuito deste trabalho é abordar, com o auxílio do instrumental da teoria dos jogos, dois instrumentos de política comercial tradicionais, as tarifas e as quotas. Os objetivos fundamentais são demonstrar que os modelos de comércio exterior com tarifas e quotas possuem estruturas que os candidatam a serem tratados como jogos finitamente repetidos e, a partir daí, tecer considerações sobre a equivalência ou não.entre tarifas e quotas.

A teoria do comércio internacional nos fornece um resultado tradicional que conclui pela equivalência. Em sua formulação inicial, ${ }^{1}$ ela significa o seguinte: o estabelecimento de uma tarifa sobre um bem importado por um país resulta num determinado volume de importações deste bem. Se, alternativamente, o país impõe uma quota igual a este volume resultante, surge uma tarifa implícita, ou seja, uma diferença entre os preços internos e externos, igual à tarifa explícita original. A recíproca também é verdadeira.

Nossa análise procura rever esta questão no contexto resultante da utilização de jogos finitamente repetidos. ${ }^{2}$ A chave da questão está na existência ou não de mais de um equilíbrio de Nash do jogo estático (i.e., o jogo de um só período). No jogo de tarifas, existem pelo menos dois, o que nos possibilita obter equilíbrios perfeitos em subjogos do jogo finitamente repetido, que não a mera repetição do equilibrio de Nash estático. Já o jogo de quotas apresenta somente

${ }^{1}$ Esta formulação inicial é devida a Bhagwati (1965).

2 Jogos repetidos enquadram-se na categoria de jogos não-cooperativos. Não estaremos lidando aqui com jogos cooperativos. 
um, o que nos deixa apenas o equilíbrio de Nash estático repetido com a característica de perfeição em subjogos.

Esses resultados estão presentes na literatura sobre teoria dos jogos aplicada ao comércio internacional e são bastantes conhecidos. $^{3}$ De fato, eles já descartam de antemão a possibilidade de serem obtidos, no jogo de quotas, equilíbrios perfeitos em subjogos Paretosuperiores à simples repetição do equilíbrio de Nash estático, já que, como frisamos acima, esta repetição constitui-se no único equilíbrio do jogo repetido. Como no jogo de tarifas, há esta possibilidade de alcançar equilíbrios Pareto-superiores, parece não haver necessidade de análises adicionais sobre a questão. Ou seja, parece já estar explícita, a esta altura, a conclusão de que não há equìvalência entre tarifas e quotas neste contexto. Entretanto, não é possível chegar a essa conclusão sem demonstrar que esses equilíbrios Pareto-superiores efetivamente fazem parte do conjunto de soluções do jogo de tarifas, o que exige a formalização do problema. Isto já foi feito no contexto de jogos finitamente repetidos com utilidades (payoffs) descontadas, onde os resultados dependem da taxa de desconto escolhida. Ou seja, é demonstrada a possibilidade de obtenção de equilíbrios Paretosuperiores dadas taxas de desconto suficientemente baixas, onde a determinação do que são taxas suficientemente baixas depende das especificações das funções utilidade dos jogadores.

A partir desta constatação, procuramos, neste trabalho, formalizar os jogos de tarifas e quotas num contexto de utilidades médias, onde a taxa de desconto, obviamente, já está determinada. As condições necessárias para a obtenção de equilíbrios perfeitos são, como veremos, mais difíceis de satisfazer e o instrumental matemático é um pouco mais sofisticado, mas os resultados obtidos apresentam a vantagem de não dependerem da especificação das funções utilidade.

Cumpre enfatizar que optamos por utilizar como funções utilidade dos jogadores (países), funções de utilidade social, o que se justifica pela ampla predominância desta escolha nos textos que aplicam jogos à teoria do comércio internacional e pelo seu próprio apelo lógico, já que é muito mais indicado tratar de políticas governamentais decididas a partir de objetivos de maximização da utilidade da

${ }^{3}$ A referência fundamental sobre este assunto é Dixit (1987). 
sociedade do que de maximização de lucros das empresas, por exemplo.

Esperamos que este trabalho possa fornecer subsídios à análise de outras formas de política comercial, como barreiras não-alfandegárias, bem como ao debate em torno destas questões, alimentado atualmente pelas perspectivas pouco favoráveis da rodada Uruguai do GATT.

2. Os modelos gerais de tarifas e quotas e a equivalência no caso estático.

O modelo que utilizamos provém da aplicação do instrumental microeconômico da teoria da dualidade ao comércio internacional, sendo, por conseqüência, bastante conhecido.

O conceito fundamental é o de função exportações líquidas ou excesso de oferta, dado por:

$$
s(p, v)=y(p, v)-z(p, v),
$$

onde $p$ é o vetor de preços, $v$ é o vetor de dotações de fatores, $y$ é a função oferta agregada e $z$ é a função demanda agregada. ${ }^{4}$

A preocupação de cada país é maximizar sua função utilidade social, cliamada de função utilidade do comércio indireta. ${ }^{5}$ Ela é dada por:

$$
H(p, r, v)=\max _{s . a .} \quad p_{s s+r \geq 0} U(s, v),
$$

onde $r=\left(p^{*}-p\right) s(p, v)$ é a renda proveniente da arrecadação tributária, sendo $p^{*}$ o vetor de preços internacionais. ${ }^{6}$ É fácil perceber que $H(p, r, v)=V(p, G(p, v)+r)$, a função utilidade social indireta, onde $G(p, v)$ é a função produto nacional máximo, ou seja:

$$
G(p, v)=\max _{\text {s.a. }} \operatorname{yy}_{\varepsilon \in(v)} p y
$$

${ }^{4}$ As definições destas funções podem ser encontradas em Woodland (1982), caps. 2,3 e 6 .

${ }^{5}$ Ver Woodland, op. cit., p. 170

${ }^{6} \mathrm{O}$ asterisco sempre indicará que a variável se refere à economia estrangeira. 
sendo $Y(v)$ o conjunto de possibilidades de produção.

Supõe-se equilíbrio no mercado internacional, ou seja,

$$
s_{i}(p, v)+s_{i}^{*}\left(p, v^{*}\right)=0, i=1, \ldots, M,
$$

onde $M$ é o número de bens. Isto equivale à definição de equilíbrio Walrasiano para $p_{i}>0$.

Sendo assim, o problema da nação doméstica é maximizar

$$
H\left(p, v, p^{*}, v^{*}\right)=V(p, m)
$$

onde a renda disponível $m=G(p, v)+\left(p-p^{*}\right) s^{*}\left(p^{*}, v^{*}\right)=G(p, v)+$ $p s^{*}\left(p^{*}, v^{*}\right)$, pois $p^{*} s^{*}\left(p^{*}, v^{*}\right)=0$ para todo $p^{*}{ }^{7}$

No modelo de quotas, o problema de maximização que precisamos consider ar, no caso geral, é da seguinte forma:

$$
\max _{\substack{p, p^{*} \\ R s=q}} V(p, m)=V\left(p, G(p, v)+\left(p-p^{*}\right) s^{*}\right)
$$

onde $R=\left(\begin{array}{ll}O \quad I\end{array}\right)$ é uma matriz e $q=\left(\begin{array}{ll}0 & q^{2}\end{array}\right)$ é um vetor, $q^{2}$ representando a quota imposta. A condição de equilíbrio no mercado mundial já foi incorporada.

O que a restrição do problema nos indica é que parte das exportações e importações estão submetidas a quotas quantitativas, ou melhor, que há uma partição dos bens em duas classes, uma em que as exportações líquidas estão submetidas a quotas, denotada por $s^{2}$, e outra em que isto não ocorre. O modelo é geral o bastante para permitir qua as exportações líquidas de todos os bens sejam restringidas por quotas.

Dados os modelos, podemos aludir à equivalência entre tarifas e quotas no caso estático como formulada pela teoria tradicional, onde estático significa que estamos limitados a um determinado período de tempo, ou seja, nossas conclusões não valem para vários períodos de tempo.

\footnotetext{
${ }^{7}$ Isto nada mais é do que a Lei de Walras.
} 
Consideremos o modelo de quotas a partir das seguintes equações:

$$
\begin{aligned}
& R s=q \\
& m=G(p, v)-\left(p-p^{*}\right) s \\
& s=G_{p}(p, v)-z(p, m) \\
& X=s+s^{*}\left(p^{*}, v^{*}\right)=0, \quad p^{1}=p^{1 *}
\end{aligned}
$$

onde $G_{p}(p, v)=\partial G(p, v) / \partial p=y(p, v),\left(p-p^{*}\right)$ é a diferença entre preços domésticos e internacionais, ocasionada pela introdução da quota, ou seja, o lucro dos exportadores e importadores e $p^{1}=p^{1 *}$ significa que os preços domésticos e internacionais daqueles bens não submetidos a quotas (que estão dentro da classe $s^{1}$ de exportações líquidas livres) devem coincidir.

Ao resolvermos o modelo, obteremos uma tarifa implícita $t=$ $p-p^{*}$ (tarifa unitária) satisfazendo a restrição imposta pela quota. Pela estrutura do modelo, é fácil perceber que, se substituirmos este valor na equação que corresponde à renda disponível $m$, sem estabelecermos o valor da quota, obteremos como solução um nível de exportações líquidas igual ao estabelecido inicialmente pela quota. $\mathrm{O}$ caminho inverso também vale, ou seja, a partir de uma tarifa explícita encontramos um nível de quota que, se colocada no lugar da tarifa, daria origem a uma tarifa implícita igual à tarifa explícita original. Daí a conclusão de equivalência entre tarifas e quotas no caso estático.

\section{Os equilinbrios de Nash estáticos do jogo de tarifas.}

O problema de maximização (5) apresenta as seguintes condições de primeira ordem: a) $s(p, v)+s^{*}\left(p^{*}, v^{*}\right)=0$; b) $p s_{p}^{*}\left(p^{*}, v^{*}\right)=0$, supondo que os preços domésticos e externos são positivos, suposição que será mantida para os demais problemas de maximização a serem resolvidos. $^{8}$

\footnotetext{
${ }^{8}$ Estes resultados podem ser encontrados em Woodland, op. cit., cap. 9. Os resultados desta seção são aplicações dos resultados de Woodland (caps. 9 e 11) para o caso de dois países e dois bens, sendo que, na análise do modelo de quotas, a dedução das curvas de reação não está presente em Woodland.
} 
A condição a) é simplesmente a restrição de equilíbrio no comércio internacional. A condição b) indica que o vetor de preços domésticos deve ser ortogonal à curva de oferta da economia estrangeira. ${ }^{9}$

Este é o resultado do modelo geral, que nos servirá para fazer a comparação com os resultados do modelo de quotas mais adiante.

Nossa atenção se concèntrará, entretanto, no caso de dois países e dois bens, por razões a serem discutidas mais à frente. Supomos que a nação doméstica impõe uma tarifa $t_{1}$ sobre o bem 1 e que a nação estrangeira impõe uma tarifa $t_{2}^{*}$ sobre o bem 2 .

Sendo assim, o problema relevante para a nação doméstica tornase:

$$
\begin{aligned}
\max _{t_{1}, p_{1}^{*}, p_{2}^{*}} V\left(p_{1}, p_{2}, m\right)= & V\left(p_{1}^{*}\left(1+t_{1}\right), p_{2}^{*}, G\left(p_{1}^{*}\left(1+t_{1}\right), p_{2}^{*}, v\right)+\right. \\
& +t_{1} p_{1}^{*} s_{1}^{*}
\end{aligned}
$$

onde já incorporamos a restrição de equilíbrio no comércio mundial e as relações entre preços domésticos e externos. O subscrito $i, i=1,2$, refere-se ao bem $i$.

As condições de primeira ordem são: ${ }^{10}$

a)

$$
\begin{aligned}
& V_{p_{1}}\left(p_{1}^{*}\right)+V_{m}\left(G_{p 1} p_{1}^{*}+p_{1}^{*} s_{1}^{*}\right)=0 \Rightarrow \\
& \Rightarrow-z_{1} p_{1}^{*}+G_{p 1} p_{1}^{*}+p_{1}^{*} s_{1}^{*}=0 \Rightarrow \\
& \Rightarrow s_{1} p_{1}^{*}+s_{1}^{*} p_{1}^{*}=0 \Rightarrow \\
& \Rightarrow p_{1}^{*}\left(s_{1}+s_{1}^{*}\right)=0
\end{aligned}
$$

b)

$$
\begin{aligned}
& V_{p 1}\left(1+t_{1}\right)+V_{m}\left(G_{p 1}\left(1+t_{1}\right)+t_{1} s_{1}^{*}+t_{1} p_{1}^{*}\left(\partial s_{1}^{*} / \partial p_{1}^{*}\right)\right)=0 \Rightarrow \\
& \Rightarrow-z_{1}\left(1+t_{1}\right)+G_{p 1}\left(1+t_{1}\right)+t_{1} s_{1}^{*}+t_{1} p_{1}^{*}\left(\partial s_{1}^{*} / \partial p_{1}^{*}\right)=0 \Rightarrow \\
& \Rightarrow s_{1}\left(1+t_{1}\right)+t_{1} s_{1}^{*}+t_{1} p_{1}^{*}\left(\partial s_{1}^{*} / \partial p_{1}^{*}\right)=0 \Rightarrow \\
& \Rightarrow s_{1}+t_{1} p_{1}^{*}\left(\partial s_{1}^{*} / \partial p_{1}^{*}\right)=0 \Rightarrow \\
& \Rightarrow t_{1}=\frac{s_{1}^{*}}{p_{1}^{*}\left(\partial s_{1}^{*} / \partial p_{1}^{*}\right)}
\end{aligned}
$$

9 Esta curva de oferta é aquela tradicional dos textos de teoria do comércio internacional.

${ }^{10}$ As funçōes aparecem sem seus argumentos por questão de economia de símbolos. Ex: $s_{1}\left(p_{1}, p_{2}, v\right)$ aparece como $s_{1}$. 
c)

$$
\begin{aligned}
& V_{p 2}+V_{m}\left(G_{p 2}+t_{1} p_{1}^{*}\left(\partial s_{1}^{*} / \partial p_{2}^{*}\right)=0 \Rightarrow\right. \\
& \Rightarrow t_{1}=\frac{s_{2}^{*}}{p_{1}^{*}\left(\partial s_{1}^{*} / \partial p_{2}^{*}\right)}
\end{aligned}
$$

A condição a) nos dá novamente a restrição de equilíbrio no comércio mundial. A condição b) nos indica que a tarifa a ser imposta pela nação doméstica para maximizar sua função de utilidade social deve ser igual ao inverso da elasticidade da oferta de exportações do bem 1 da nação estrangeira, $\varepsilon_{1}^{*}$, ou seja,

$$
t_{1}=1 / \varepsilon_{1}^{*}
$$

A condição c) é marginal e sem significado analítico. Se resolvêssemos o problema análogo para a nação estrangeira, obteríamos

$$
t_{2}^{*}=1 / \varepsilon_{2 \prime}
$$

onde $\varepsilon_{2}$ é a elasticidade da oferta de exportações do bem 2 da nação doméstica.

Estas condições, entretanto, só valem para o caso em que a economia estrangeira não impõe tarifas. Vejamos o que ocorre quando existe a tarifa estrangeira.

Neste caso, as relações entre preços domésticos, estrangeiros e internacionais são dadas por

$$
p_{1}=p_{1}^{0}\left(1+t_{1}\right) ; \quad p_{2}=p_{2}^{0} ; \quad p_{1}^{*}=p_{1}^{0} ; \quad p_{2}^{*}=p_{2}^{0}\left(1+t_{2}^{*}\right),
$$

onde $p_{1}^{0}$ e $p_{2}^{0}$ são os preços do mercado mundial (internacionais).

$O$ problema de maximização torna-se

$$
\begin{aligned}
\max _{t_{1}, p_{1}^{0}, p_{2}^{0}} V\left(p_{1}, p_{2}, m\right) & =V\left(p_{1}^{0}\left(1+t_{1}\right), p_{2}^{0}, G\left(p_{1}^{0}\left(1+t_{1}\right), p_{2}^{0}, v\right)+\right. \\
& \left.+t_{1} p_{1}^{0} s_{1}^{*}\left(p_{1}^{0}, p_{2}^{0}\left(1+t_{2}^{*}\right), v\right)\right)
\end{aligned}
$$

É fácil verificar que as mesmas condições do problema anterior são requeridas. Sendo assim, podemos derivar curvas de reação para as economias doméstica e externa. 
Para cada valor da tarifa externa imposta, a economia doméstica estabelecerá sua própria tarifa, de acordo com a função de reação da nação doméstica.

$$
T\left(t_{2}^{*}\right)=1 / \varepsilon_{1}^{*}
$$

onde $\varepsilon_{1}^{*}$ é função de $t_{2}^{*}$.

Para a economia estrangeira, a função

$$
T^{*}\left(t_{1}\right)=1 / \varepsilon_{2}
$$

dá a sua reação a diferentes níveis da tarifa doméstica, já que $\epsilon_{2}$ é função de $t_{1}$.

Podemos ilustrar este processo graficamente. A figura 1 abaixo nos dá as curvas de oferta da economia doméstica, designadas por OD, e as da economia estrangeira, designadas por OE. As curvas de indiferença II são domésticas e as $I^{*} I^{*}$, estrangeiras. Como de costume, supõe-se a possibilidade de agregação das preferências individuais, de tal forma que as curvas de indiferença, com as características habituais de convexidade e não-intersecção, surgem de uma função de utilidade social.

Ao instituir a tarifa ótima $t_{1}$ sobre as suas importações do bem 1, a economia doméstica apresenta uma nova curva de oferta $O D^{1}$, que intercepta a curva $O E^{0}$ no ponto $P^{\prime}$, que corresponde ao ponto cuja reta tangente tangencia a curva de indiferença de maior utilidade possível $I^{2} I^{2}$. Em seguida, a economia estrangeira retalia, instituindo uma tarifa sobre o bem 2, por ela importado, de tal forma a maximizar sua utilidade, o que ocorre quando sua curva de oferta se torna $O E^{1}$. Isto fica claro quando verificamos que o ponto de interseção de $O E^{1}$ com $O D^{1}, P^{\prime \prime}$, é o ponto de tangência de $O D^{1}$ com $I^{* 2} I^{* 2}$, a curva de indiferença de maior utilidade que pode atingir.

$P^{\prime}$ e $P^{\prime \prime}$ estão sobre as curvas de reação da economia doméstica e da economia estrangeira, respectivamente. Estas curvas de reação nada mais são do que loci dos pontos de tangência entre as curvas de indiferença de mais alta utilidade de um país com as curvas de oferta correspondentes do outro país. A figura 2 nos mostra uma curva de reação para cada país, derivada a partir das curvas de oferta. ${ }^{11} O R^{1}$

${ }^{11}$ Frisamos que estas são apenas possíveis conformaçōes para as curvas de reação. 


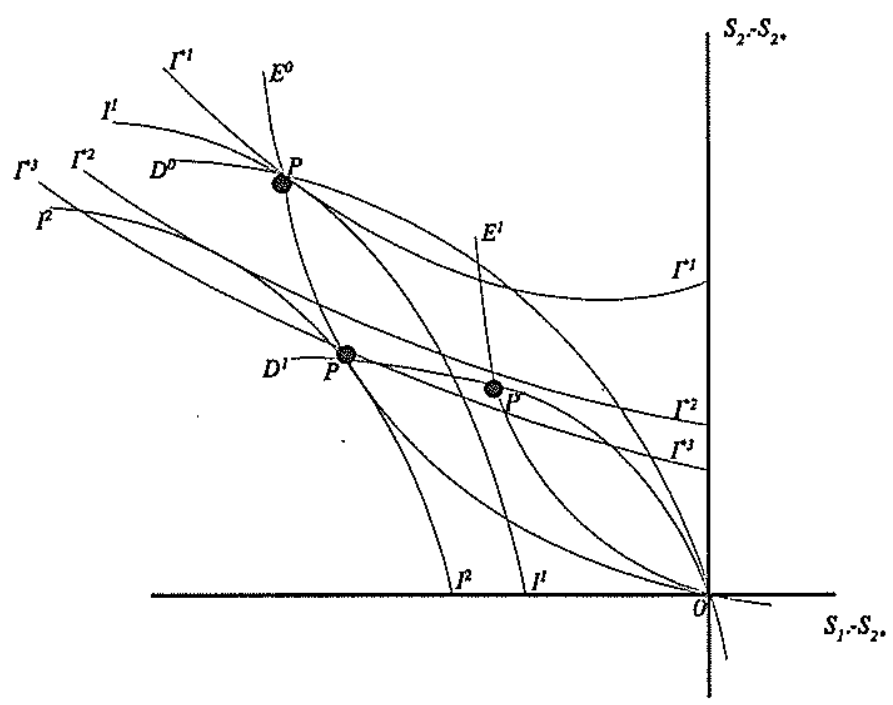

Figura 1.

nos dá os pontos de tarifa ótima da nação doméstica para cada tarifa imposta pela nação estrangeira. $O R^{2}$ cumpre a mesma função para a economia estrangeira.

Procuremos então o equilíbrio de Nash do jogo de tarifas estático. Sabemos que ele surge quando as equações (16) e (17) são satisfeitas simultaneamente, isto é, quando as curvas de reação dos dois países se interceptam. ${ }^{12}$ Sendo assim, é preciso que $T\left(t_{2}^{*}\right)=T^{*}\left(t_{1}\right)$, o que significa, utilizando (10) e (13):

$$
\frac{s_{1}^{*}}{p_{1}^{*}\left(\partial s_{1}^{*} / \partial p_{1}^{*}\right)}=\frac{1}{s_{2} /\left(p_{2}\left(\partial s_{2} / \partial p_{2}\right)\right)}
$$

ou

$$
s_{1}^{*} s_{2}=\left(p_{1}^{*}\left(\partial s_{1}^{*} / \partial p_{1}^{*}\right)\right)\left(p_{2}\left(\partial s_{2} / \partial p_{2}\right)\right)
$$

$12 \mathrm{O}$ raciocínio é o mesmo daquele aplicado à obtenção do equilíbrio de Nash num modelo de oligopólio. 


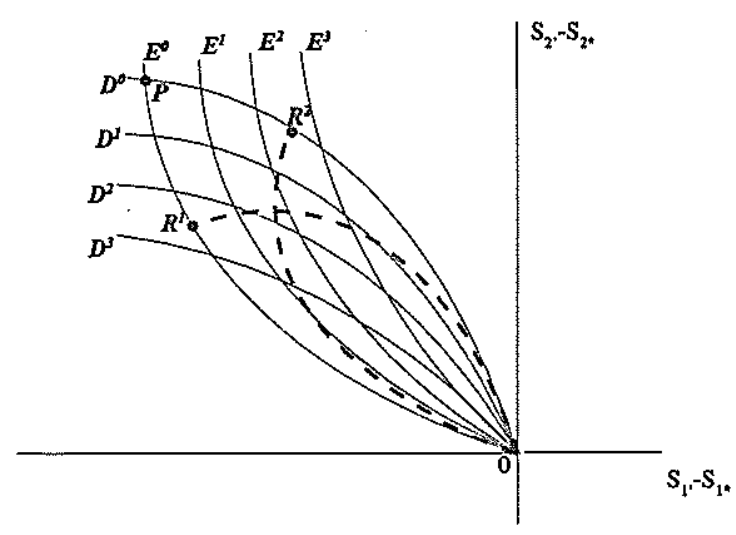

Figura 2.

Precisamos provar que este equilíbrio existe. ${ }^{13} \mathrm{Em}$ primeiro lugar, sabemos que $s_{1}^{*}\left(p^{*}, r^{*}, v^{*}\right)=s_{1}^{*}\left(p^{*}, G^{*}\left(p^{*}, v^{*}\right)+t_{2}^{*} p_{2} s_{2}, v^{*}\right), p^{*}=$ $\left(p_{1}^{*}, p_{2}^{*}\right)$, ou seja, que $s_{1}^{*}$ é função de $t_{2}^{*}$. Dessa forma, dada a tarifa $t_{2}^{*}$, existe uma única tarifa $t_{1}$ associada, como pode-se perceber imediatamente ao analisar (16). Igualmente, para cada tarifa $t_{1}$ há uma única tarifa $t_{2}^{*}$ associada.

Em segundo lugar, o $t_{1}$ inicial, ou seja, a tarifa doméstica ótima, calculada a partir da situação de livre comércio, deve ser positivo, o que ocorre pelo fato de que sempre há um ganho de utilidade para o país quando ele, a partir de um cenário de tarifas nulas, passa a impor uma tarifa. Mais uma vez, o mesmo vale para o $t_{2}^{*}$ inicial.

Em terceiro lugar, a curva de reação do país deve passar por algum ponto verticalmente acima do $t_{2}^{*}$ inicial do outro país. Isto indica que, para $t_{1}=0$, temos $T^{*}\left(t_{1}\right)=t_{2}^{*^{0}}$ na curva de reação da economia estrangeira e correspondendo a $t_{2}^{*^{0}}, T\left(t_{2}^{*^{0}}\right)>0$ na curva de

${ }^{13}$ Esta prova segue as indicações fornecidas por Johnson (1953/54). Embora àquela época o instrumental de teoria dos jogos não estivesse disponível, a análise de Johnson corresponde a uma busca do equilíbrio de Nash do jogo estático. 
reação da economia doméstica. Isto ocorre porque

$$
T\left(t_{2}^{*}\right)=\frac{s_{1}^{*}}{p_{1}^{*}\left(\partial s_{1}^{*} / \partial p_{1}^{*}\right)}=0 \Rightarrow s_{1}^{*}=0,
$$

ou seja, não há comércio e

$$
s_{1}^{*}\left(p^{*}, G^{*}\left(p^{*}, v^{*}\right)+t_{2}^{*} p_{2} s_{2}, v^{*}\right) \geq 0
$$

por hipótese. Ou seja, $T\left(t_{2}^{*}\right)$ só seria igual a zero se não houvesse comércio. Como $T^{*}\left(t_{1}=0\right)$ não é uma tarifa proibitiva, ou seja, que conduza à interrupção do comércio, concluímos que $T\left(t_{2}^{*^{0}}\right)>0$. A curva de reação da nação estrangeira, como de praxe, observa a mesma condição.

Em último lugar, sabemos que $T\left(t_{2}^{*}\right)=0$ na curva de reação doméstica implica $t_{2}^{*}>t_{2}^{*}$, ou seja, a curva de reação doméstica intercepta o eixo dos $t_{2}^{*}$ em um ponto além de $t_{2}^{*^{0}}$. A prova disso é que, no nosso problema de maximização, a tarifa doméstica ótima sempre será positiva à exceção de quando não há comércio, isto é, quando a nação estrangeira estabelece uma tarifa $t_{2}^{*}$ tão alta que elimina as transaçōes internacionais. Como $t_{2}^{*^{0}}$ não elimina o comércio, como mencionamos acima, concluímos que o $t_{2}^{*}$ correspondente a $T\left(t_{2}^{*}\right)=0$ é maior do que $t_{2}^{*^{0}}$.

Estas consideraçōes garantem a existência de ao menos um ponto de interseção das curvas de reação dos dois países, isto é, de um equilíbrio de Nash do jogo estático. ${ }^{14}$

Há outro equilíbrio de Nash do jogo estático de tarifas que não resulta da análise desenvolvida acima. Trata-se do equilíbrio de Nash autárquico, ${ }^{15}$ ou seja, aquele que surge da imposição por um dos países, ou por ambos, de uma tarifa tão alta que iniba inteiramente o comércio. Esta situação configura um equilíbrio de Nash porque não importa o nível de tarifas que um dos países imponha, o resultado

\footnotetext{
${ }^{14}$ Esta análise das curvas de reação poderia ter sido feita em termos gráficos, como pode ser encontrado em Johnson, op. cit. Entretanto, procuramos desenvolvê-lạ em termos do instrumental microeconômico moderno, o que nos parece conferir maior força ao argumento.

${ }^{15}$ A existência deste equilíbrio foi percebida pela primeira vez por Dixit (1987).
} 
será sempre a ausência de comércio, pois o outro país está administrando uma tarifa proibitiva. Sendo assim, nenhum dos países pode aumentar seu nível de utilidade através da alteração de sua política tarifária.

Chamando de $p_{a}$ e $p_{a}^{*}$ os preços relativos do bem 1 com relação ao 2 que prevaleceriam na economia doméstica e na estrangeira, respectivamente, caso não houvesse comércio entre elas, obteríamos a seguinte equação que define os níveis de tarifas proibitivas:

$$
\left(1+t_{1}\right)\left(1+t_{2}^{*}\right)=p_{a} / p_{a}^{*}
$$

Portanto, podemos concluir que o jogo de tarifas estático tem ao menos dois equilíbrios de Nash, o que será fundamental para o desenvolvimento posterior da análise.

\section{O equilíbrio de Nash estático do jogo de quotas.}

Nossa tarefa agora é fazer o mesmo tipo de análise para o caso de quotas, a qual perseguiremos seguindo os mesmos passos da análise de tarifas. ${ }^{16}$

O problema de maximização que precisamos considerar, no caso geral, é aquele dado por (6). Conduziremos a análise em termos deste problema por motivos operacionais, considerando que não há nenhum prejuízo para a comparação com o caso de tarifas. Entretanto, podemos passar a trabalhar logo com o problema que supõe já haver quotas estabelecidas pelo outro país. Este problema fica da seguinte forma:

$$
\max _{\substack{p, p^{*} \\ \text { s.a. } \\ R^{*} s^{*}=q^{*}}} V(p, m)=V\left(p, G(p, v)+\left(p-p^{*}\right) s^{*}\right),
$$

onde $R=\left(\begin{array}{ll}O & I\end{array}\right)$ e $R^{*}=\left(\begin{array}{ll}O & I^{*}\end{array}\right)$ são matrizes, sendo que $\mathrm{R}$ pode diferir de $R^{*}$, e $q^{*}=\left(\begin{array}{ll}0 & q^{2 *}\end{array}\right)$ é um vetor de quotas da nação estrangeira.

\footnotetext{
${ }^{16}$ Esta seção baseia-se em Tower (1975), cujos resultados demonstram que o jogo de quotas só apresenta um equilíbrio de Nash estático, qual seja o equilíbrio autárquico, embora esta linguagem de teoria dos jogos não esteja presente em seu trabalho. Outra referência importante é Rodriguez (1974), que chegou aos meus resultados de maneira independentemente.
} 
Seu Lagrangeano é

$$
L=V\left(p, G(p, v)+p s^{*}\right)+\lambda\left(q+R s^{*}\right)+\lambda^{*}\left(q^{*}-R^{*} s^{*}\right),
$$

onde $\lambda=\left(\lambda_{1}, \ldots, \lambda_{M}\right)$ é o vetor de multiplicadores de Lagrange da restrição doméstica e $\lambda *$, o da estrangeira.

As condições de primeira ordem nos informam que

a)

$$
\begin{aligned}
& V_{p}+V_{m}\left(G_{p}+s^{*}\right)=0 \Rightarrow \\
& \Rightarrow-z+G_{p}+s^{*}=0 \Rightarrow \\
& \Rightarrow s+s^{*}=0
\end{aligned}
$$

b)

$$
V_{m}\left(p s_{p}^{*}\right)+\lambda R s_{p}^{*}-\lambda^{*} R^{*} s_{p}^{*}=0
$$

c)

$$
\begin{aligned}
& q+R s^{*}=0 \Rightarrow \\
& \Rightarrow R s=q
\end{aligned}
$$

d)

$$
\begin{aligned}
& q^{*}-R^{*} s^{*}=0 \Rightarrow \\
& \Rightarrow R^{*} s^{*}=q^{*}
\end{aligned}
$$

As condiçōes c) e d) repetem as restriçōes do problema de maximização. A condição a) mais uma vez refere-se ao equilíbrio comercial mundial. A condição b) passaremos a apreciar agora.

Em primeiro lugar, sabemos que para maximizar $\bar{V}$, o valor ótimo da nossa função objetivo, com relação a $q$, devemos ter $\lambda=0$, o que dá à condição b) a nova configuração:

$\left.b^{\prime}\right)$

$$
V_{m}\left(p s_{p}^{*}\right)-\lambda^{*} R^{*} s_{p}^{*}=0
$$

Mas ela ainda pode ser simplificada se percebermos que $R^{*} s_{p}^{*}=$ 0 , pela diferenciação da condição d). Passamos a ter então 
$\left.b^{\prime \prime}\right)$

$$
\begin{aligned}
& V_{m}\left(p s_{p}^{*}\right)=0 \Rightarrow \\
& \Rightarrow p s_{p}^{*}=0,
\end{aligned}
$$

que é exatamente igual à condição b) para a maximização do problema (5). ${ }^{17}$

Estamos aptos agora a tecer consideraçōes sobre a existência ou não de equilíbrios de Nash estático do jogo de quotas. A quota ótima a ser estabelecida pela economia doméstica, a partir do livre comércio ou de uma situação em que o outro país já impõe uma quota, determina uma tarifa implícita $t_{1}=1 / \varepsilon_{1}^{*}$, igual à tarifa ótima explícita. Para a nação estrangeira, temos a mesma situação, sendo que a tarifa implícita é $t_{2}^{*}=1 / \varepsilon_{2}$. Entretanto, ao contrário do jogo de tarifas, as curvas de reação dos dois países não se encontram, ${ }^{18}$ como veremos a seguir.

Para $M=2$, as condições de primeira ordem do problema de maximização da utilidade sob regime de quotas para a nação doméstica tornam-se:

a)

$$
s_{1}+s_{1}^{*}=0 ; \quad s_{2}+s_{2}^{*}=0
$$

b)

$$
\begin{aligned}
& p_{1}\left(\partial s_{1}^{*} / \partial p_{1}^{*}\right)+p_{2}\left(\partial s_{2}^{*} / \partial p_{1}^{*}\right)=0 \\
& p_{1}\left(\partial s_{1}^{*} / \partial p_{2}^{*}\right)+p_{2}\left(\partial s_{2}^{*} / \partial p_{2}^{*}\right)=0
\end{aligned}
$$

c)

$$
\begin{aligned}
& R=\left(\begin{array}{ll}
1, & 0
\end{array}\right), \quad q \text { escalar } \\
& R s=q \Rightarrow(1, \quad 0)\left(\begin{array}{l}
s_{1} \\
s_{2}
\end{array}\right)=q \Rightarrow s_{1}=q
\end{aligned}
$$

${ }^{17}$ Podemos obter os mesmos resultados dos problemas de maximização (8) e (15) a partir das condições a) e b) do problema (5).

${ }^{18} \mathrm{~A}$ situação, e m termos da análise tradicional de curvas de oferta, é a seguinte: ao procurar maximizar sua utilidade, cada país se defronta com curvas de oferta do outro país cada vez mais restritas, porém mantendo a mesma configuração abaixo do nivel da quota. Sendo assim, fica evidente que a satisfação da condição (27) só se pode dar a um nível de quota inferior ao anteriormente estabelecido, o que mostra uma tendência a zero das quotas. 
d)

$$
\begin{aligned}
& R^{*}=\left(\begin{array}{ll}
0 & 1
\end{array}\right), q^{*} \text { escalar } \\
& R^{*} s^{*}=q^{*} \Rightarrow\left(\begin{array}{ll}
0 & 1
\end{array}\right)\left(\begin{array}{l}
s_{1}^{*} \\
s_{2}^{*}
\end{array}\right)=q^{*} \Rightarrow s_{2}^{*}=q^{*}
\end{aligned}
$$

Sendo assim, dado $q^{*}$, o país tem que estabelecer uma quota $q$ tal que estas condições sejam satisfeitas. Como o outro país já estipulou sua quota, temos que $s_{2}=-q^{*}$ no equilíbrio anterior. Portanto, a nação doméstica tem que estipular uma quota que produza um vetor de preços $p$ tal que as condições (28) sejam satisfeitas por um $s_{2}$ menor do que $q^{*}$. Ora, isto só pode ocorrer se $q$ for fixado abaixo do $s_{1}$ inicial. Portanto, o processo tende ao fim do comércio. Qualquer que seja o nível de quota imposto pelo outro país, é melhor, para o país que está tentando maximizar sua utilidade, estipular uma quota que não aquela que prevalecia anteriormente. Esta situação, obviamente, indica que não há uma combinação de quotas em que ambos os países estejam satisfeitos com sua condição, ou seja, em que ambos julguem que agindo unilateralmente não poderão obter ganhos de utilidade. Portanto, não há um ponto que seja um equilíbrio de Nash para quotas positivas.

Vejamos como esta análise se desenvolve em termos gráficos. $\mathrm{Na}$ figura 3, abaixo, supomos que a nação doméstica impõe uma quota. Como a curva de indiferença de maior utilidade que pode ser atingida por esta nação sobre a curva de oferta da nação estrangeira $\left(O E^{0}\right)$ é II, ela estabelecerá a quota $O A_{1}$ sobre o bem que importa (bem 1), fazendo com que sua curva de oferta se torne $O A_{1}{ }^{\prime} P_{1} A_{1}$. A interseção das duas curvas de oferta passa a ocorrer no ponto $P_{1}$.

A nação estrangeira tem agora que estabelecer uma quota ligeiramente inferior a $O P_{1}^{\prime}$, caso contrário o sistema permanecerá no ponto $P_{1}$. Digamos que ela estabeleça $O B_{1}$, o que lhe permitirá obter a maior utilidade possível, dada a restrição estabelecida pela quota do outro país sobre suas (da nação doméstica) importações. Dessa forma, sua curva de oferta será $O B_{1}^{\prime} B B_{1}$, e o sistema passa a repousar no ponto $B$.

Pelo mesmo raciocínio do parágrafo anterior, a política ótima para a nação doméstica passará a ser o estabelecimento da quota 


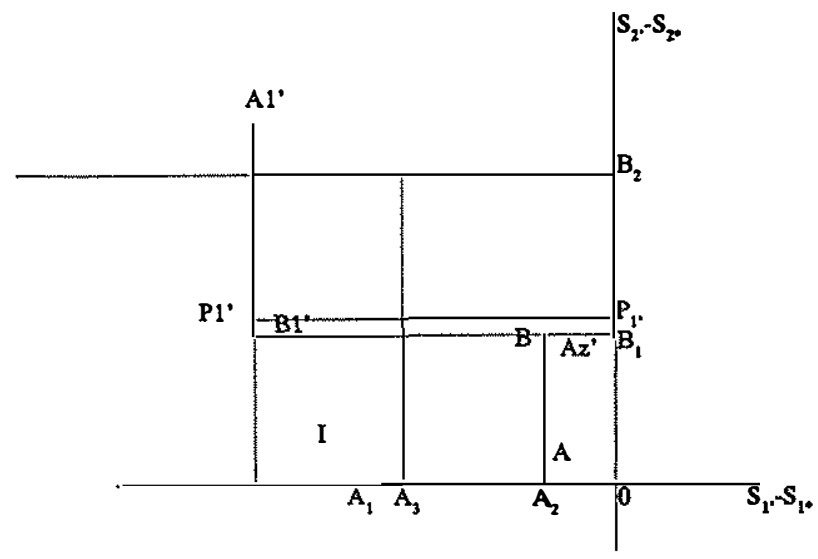

Figura 3.

$O A_{2}$, de tal forma que sua curva de oferta passe a ser $O A_{2}{ }^{\prime} A A_{2}$ e as curvas de oferta se interceptem no ponto $A$.

A continuidade deste processo levaria o sistema a aproximar-se assintoticamente da autarquia, ou seja, da eliminação do comércio. Isto ilustra a nossa afirmação de que não há um ponto que seja um equilíbrio de Nash para quotas positivas.

$\mathrm{O}$ leitor atento já deve ter verificado que o fato de nos termos restringido a quotas positivas acima se justifica porque com quotas de valor zero entramos na situação de autarquia, que, pelas mesmas razões apontadas para o caso de tarifas, constitui um equilíbrio de Nash estático. Sendo assim, concluímos que o jogo de quotas apresenta um equilíbrio de Nash estático, mas ele é único.

\section{Jogos finitamente repetidos de tarifas e quotas.}

Os trabalhos existentes sobre a aplicação de teoria dos jogos aos modelos de tarifas e quotas já estabeleceram a existência de equilíbrios perfeitos em subjogos em jogos infinitamente repetidos de tarifas. ${ }^{19}$

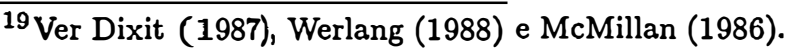


Sendo assim, concentraremos nossa atenção em jogos finitamente repetidos, embora não pareça haver na literatura estudos referentes a jogos infinitamente repetidos de quotas, o que poderia ser objeto de consideraçōes.

Os teoremas e lemas principais de jogos finitamente repetidos, em particular os que utilizaremos a seguir, exigem a existência de ao menos dois equilíbrios de Nash do jogo estático. Sendo assim, resulta imediata a constatação de que o jogo finitamente repetido de quotas deve apresentar problemas no que se refere à obtenção de equilíbrios perfeitos em subjogos.

Para que possamos fazer uma comparação efetiva entre tarifas e quotas neste contexto, precisamos mostrar que o jogo de tarifas não apresenta os mesmos problemas, o que não é garantido pela simples existência de dois equilíbrios de Nash estáticos deste jogo.

Comecemos por apresentar, de forma bastante informal ${ }^{20}$, os resultados de um teorema fundamental de jogos finitamente repetidos com utilidades descontadas. Este teorema já foi utilizado na literatura para demonstrar a possibilidade de o jogo de tarifas apresentar equilíbrios perfeitos em subjogos Pareto-superiores à simples repetição do equilíbrio de Nash estático. Antes disto, porém, precisamos introduzir algumas notações.

Chamaremos de $G(T)$ o jogo finitamente repetido com duração de $T$ períodos, sendo que há $n$ jogadores neste jogo. Usaremos $a=\left(a_{1}, \ldots, a_{n}\right)$ para indicar uma combinação de estratégias dos $n$ jogadores e $P_{i}(a) \quad \epsilon \quad R$ para representar o utilidade do jogador $i$ associado à combinação $a$. Alex́n disso, ${ }^{i} a$ representará o pior equilíbrio de Nash do jogo de um só período para o jogador $i, a^{0}$ outro equilíbrio de Nash deste jogo tal que $P_{i}\left(a^{0}\right)>>P_{i}\left({ }^{i} a\right)$ para todo $i$ (isto é, cada jogador prefere este equilíbrio ao seu pior equilíbrio de Nash) e $a^{*}$ um determinado resultado do jogo estático a ser jogado por várias rodadas tal que $P_{i}\left(a^{*}\right)>>P_{i}\left({ }^{i} a\right), \quad i=1, \ldots, n$. Por fim, $b_{i}(a)$ é a

\footnotetext{
${ }^{20} \mathrm{~A}$ demonstração do teorema que vem após este já é suficientemente sofisticada para satisfazer o desejo do leitor por formalização matemática. Além disso, a apresentação detalhada deste teorema e de sua demonstração envolveria uma adição desnecessária de símbolos e definições, já que não iremos utilizá-lo na análise que se segue. O leitor interessado pode encontrar este teorema de forma completa em Friedman (1986), cap.3.
} 
melhor resposta do jogador $i$ à combinação de estratégias $a$.

A idéia do teorema é a seguinte. Os jogadores estipulam um determinado resultado do jogo estático, $a^{*}$ - estritamente preferido por todos eles aos seus respectivos piores equilíbrios de Nash - a ser jogado por várias rodadas. Se nenhum jogador $j$ desviar-se de $a^{*}$ até o período $t^{*}$, o jogador $i$ joga $a_{i}^{*}$ nos períodos $t=1, \ldots, t^{*}$ e passa a jogar $a_{i}^{0}$ para $t=t^{*}+1, \ldots, T$, desde que o jogador $j$ escolha $a_{j}^{0}$ do período $t^{*}+1$ em diante. Caso algum jogador $j$ desvie-se da trajetória $\left(a^{*}, a^{*}, \ldots, a^{*}, a^{0}, a^{0}, \ldots, a^{0}\right)$ em algum período $t$, o jogador $i$ passará a jogar $j^{\prime} a_{i}$, onde $j^{\prime}$ é o maior índice dos $j$ que se desviaram. Perceba que, a partir de determinado período, os jogadores passam a jogar repetidamente o equilíbrio de Nash $a^{0}$. Isto é imposto à estrutura do jogo pelo argumento de indução retrospectiva. ${ }^{21}$

Este tipo de estratégia, chamada de trigger strategy, permite que surjam equilinbrios perfeitos em subjogos (isto é, equilíbrios que não contenham estratégias com ameaças não-críveis) Pareto-superiores à repetição do equilíbrio de Nash estático. É isto que prova o teorema que estamos discutindo. Simplificadamente, ele diz que, em um jogo finitamente repetido $G(T)$, onde cada jogador desconta suas utilidades futuras utilizando uma taxa de desconto $\rho_{i}$, que contenha ao menos dois equilíbrios de Nash estáticos, uma combinação de trigger strategies (ou seja, cada jogador agindo de acordo com o que foi especificado pela sua trigger strategy) é um equilíbrio perfeito em subjogos se, dadas algumas outras condições, tivermos, para cada jogador $i$,

$$
\alpha_{i}>\frac{b_{i}\left(a^{*}\right)-P_{i}\left(a^{*}\right)}{b_{i}\left(a^{*}\right)-P_{i}\left(a^{*}\right)+P_{i}\left(a^{0}\right)-P_{i}\left({ }^{i} a\right)},
$$

se $P_{i}\left(a^{*}\right)>P_{i}\left({ }^{i} a\right)$, onde $\alpha_{i}=1 /\left(1+\rho_{i}\right)$, ou

$$
\alpha_{i}>\frac{b_{i}\left(a^{*}\right)-P_{i}\left(a^{*}\right)}{b_{i}\left(a^{*}\right)-P_{i}\left({ }^{i} a\right)}
$$

se $P_{i}\left(a^{*}\right) \leq P_{i}\left(a^{0}\right)$.

${ }^{21}$ Uma explicação sobre este argumento pode ser encontrada em Fuedenberg \& Tirole (1991), pp. 72-74. 
Podemos perceber que, caso estejamos interessados em saber se determinado jogo finitamente repetido possui ou não tais equilíbrios, precisamos calcular as utilidades de todos os jogadores nos pontos especificados, bem como suas melhores respostas, para então podermos comparar os resultados obtidos com os respectivos $\alpha_{i}^{\prime} s$. Ou seja, nossas conclusões dependem das funções utilidade e das taxas de desconto especificadas.

A aplicação deste teorema ao jogo de tarifas, portanto, sofre destas limitações. Sendo assim, precisamos restringirmo-nos a um jogo com taxas de desconto definidas, se quisermos obter resultados mais potentes. A escolha mais imediata é a de jogos onde não se desconta o futuro, ou seja, onde as utilidades de diferentes períodos têm o mesmo peso no cálculo da utilidade total. Esta escolha tem a vantagem de que já foi desenvolvido por Benoit \& Krishna ${ }^{22}$ o instrumental de jogos finitamente repetidos com utilidades médias, o qual utilizaremos aqui.

Feita esta escolha, no entanto, as trigger strategies acima apresentadas já não são capazes de sustentar todos os equilíbrios perfeitos em subjogos de $G(T) .{ }^{23}$ Sendo assim, precisamos encontrar estratégias com punições mais severas do que as estipuladas pelas trigger strategies. Benoit \& Krishna sugerem punições em três fases.

A idéia deste tipo de estratégia é a seguinte. Supondo que o jogador $i$ deve ser penalizado, os demais jogadores agem de tal forma a penalizá-lo fortemente numa primeira fase, jogando sua estratégia de minmax (do jogador i) por um longo período, estratégia esta que oferece aos outros jogadores $j \neq i$ incentivos para que dela se desviem. A segunda fase vem justamente oferecer compensações aos jogadores $j \neq i$ para evitar este desvio na primeira fase, com o cuidado, é claro, de evitar que o jogador $i$ recupere as suas perdas. A terceira e última fase consiste em jogar um dos equilíbrios de Nash do jogo de um só período repetidamente, o que, como já vimos, é obrigatório em um jogo finito. A duração desta fase deve ser tal que assegure a adesão de todos os jogadores à trajetória determinada para a segunda fase.

Entendido isso, o que faremos agora é provar que o jogo finita-

${ }^{22}$ Benoit \& Krishna (1985).

${ }^{23}$ Ver Benoit \& Krishna, op. cit., p. 912. 
mente repetido de tarifas com utilidades médias apresenta equilíbrios perfeitos em subjogos, demonstrando, a princípio, o seguinte lema, ${ }^{24}$ que formaliza o mecanismo para construir este tipo de punições em três fases para o jogo de tarifas.

Lema 1. Suponha que um jogo finitamente repetido de tarifas $G(T)$ consista de dois jogadores (paises). Chame de $a^{j 0}$ um equilíbrio de Nash não-autárquico do jogador $j$, de $P_{j}\left(a^{j 0}\right)$ e $w_{j}(1)$ as utilidades associadas a este equilíbrio e ao equilíbrio autárquico, respectivamente, para $j=1,2$. Faça $r=(r, \ldots, r)$, um vetor de constantes e $y_{j}^{i}$ representar o payoff do jogador $j \neq i$ quando o jogador $i$ é submetido ao seu minmax. Podemos demonstrar que, se $P_{j}\left(a_{j}^{0}\right)>w_{j}(1)$, então:

a) Existe um $T_{j}$ e uma punição em $S\left(T_{j}\right)$, o conjunto dos equilíbrios perfeitos em subjogos do jogo repetido $T_{j}$ vezes, em que o payoff médio de $j$ é $z_{j}$. Além disso, $\left(z_{i}, z_{-i}+r_{-i}\right)$ pertence ao conjunto de possibilidades de utilidades, onde o subscrito $-i$ significa, em geral, os payoffs (como neste caso) ou as estratégias dos $(n-1)$ jogadores que não $i$;

b) Escolhendo $i^{\prime} \epsilon \operatorname{argmax}_{j / i}\left(z_{j}-y_{j}^{i}\right)$, existe um $\eta_{i}^{\text {T* }}$ e uma punição em $S\left(T_{i}^{*}\right)$ na qual o payoff médio do jogador $i$ é menor do que $\phi_{i}(z, r)+\omega, \omega>0$, onde $\phi_{i}(z, r)=\lambda v_{i}+(1-\lambda) z_{i} \mathrm{e}$ $\lambda=\left(r /\left(r+z_{i^{\prime}}-y_{i^{\prime}}^{i}\right) \in(0,1)\right.$.

Demonstração: Em primeiro lugar, é fácil verificar que efetivamente $P_{j}\left(a_{j}^{0}\right)>w_{j}(1)$, já que a utilidade associada ao equilíbrio autárquico é menor do que a associada ao equilíbrio não-autárquico. ${ }^{25}$ Comecemos então pela demonstração da parte a). O equilíbrio em $S\left(T_{j}\right)$ nos é fornecido pela trigger strategy adequada. Isto significa que, para cada $j$, escolhemos um equilíbrio perfeito em subjogos representado por uma trigger strategy que produz uma utilidade média maior do que $w_{j}(1)$, o que ocorre poraue sabemos que este tipo de estratégia nos possibilita atingir utilidades superiores as da simples repetição do equilíbrio de Nash estático. ${ }^{26}$ Sendo asim, temos

24 Este lema baseia-se no lema 3.5 de Benoit \& Krishna, op. cit., pg. 915

${ }^{25}$ Este é um resultado bastante conhecido na literatura (ver Dixit, op. cit.)

${ }^{26}$ Para uma definição de trigger strategy e a demonstração de teoremas a ela associados ver Friedman (1986), cap. 3 
$z_{j}>w_{j}(1)$. Entretanto, precisamos mostrar que este equilíbrio perfeito em subjogos efetivamente existe, uma vez que na literatura este resultado está estabelecido geralmente para utilidades descontadas, o que não é o nosso caso aqui, já que trabalhamos com utilidades médias. As condições a serem satisfeitas, à exceção de uma, são as mesmas do lema. Sendo assim, precisamos provar a que falta. Para que o equilíbrio ocorra, é suficiente que

$$
\begin{aligned}
& \sum_{t=0}^{t^{*}} P_{j}\left(a^{*}\right)+\sum_{t=t^{*}+1}^{T j} P_{j}\left(a^{0}\right)>\sum_{t=0}^{t 0-1} P_{j}\left(a^{*}\right)+b_{j}\left(a^{*}\right)+ \\
& +\sum_{t=t 0+1}^{T j} w_{j}(1),
\end{aligned}
$$

para todo $j$. Nesta expressão, $a^{*}$ é qualquer combinação de tarifas que conduza a uma utilidade maior do que $w_{j}(1)$, podendo inclusive ser um ponto sobre a fronteira eficiente de Pareto e $a^{0}=a^{10}=a^{20}$. Além disso, $t_{0}<t^{*}$ é o período em que o jogador $j$ se desvia da trajetória estabelecida, $t^{*}$ é o período em que há a reversão obrigatória para o equilíbrio de Nash estático $a^{0}$ e $b_{j}\left(a^{*}\right)$ é a melhor resposta a $a^{*}$ do jogador $j$.

Manipulando (31), chegamos à conclusão de que

$$
b_{j}\left(a^{*}\right)-P_{j}\left(a^{*}\right) \leq \Sigma_{t=t 0+1}^{t^{*}}\left(P_{j}\left(a^{*}\right)-w_{j}(1)\right)
$$

é uma condição suficiente para que a combinação de trigger strategies dos jogadores produza um equilíbrio perfeito em subjogos.

Sendo assim, para dados $t_{0}$ e $t^{*}$, chamamos de $\delta_{i}$ o menor valor inteiro de $T_{j}-t^{*}$ tal que a inequação (32) seja satisfeita. Sendo $\gamma=\max _{i \in N} \delta_{i}$, temos um equilíbrio perfeito do jogo se $t^{*} \geq 0 \mathrm{e}$ $T_{j} \geq t^{*}+\gamma$. Portanto, já conseguimos o nosso equilíbrio em $S\left(T_{j}\right)$.

Como o nosso jogo só tem dois jogadores, a outra parte da proposição a) decorre imediatamente do que acabamos de apresentar.

Com relação à parte b), começamos por definir as trajetórias de resultados ${ }^{27} c^{*}=\left(c^{1}, c^{2}, \ldots, c^{k}\right)$, que gera as utilidades médias $\left(z_{i}, z_{-i}+r_{-i}\right), a^{0 *}=\left(a^{10}, \ldots, a^{n 0}\right),{ }^{28}$ um ciclo de equilíbrios de Nash

${ }^{27}$ Significa a série de resultados que efetivamente ocorrem em decorrência das escolhas de estratégias dos jogadores.

${ }^{28}$ Perceba que usamos uma notação bastante geral para conduzir a demonstração. 
não-autárquicos e

$$
\left(m^{i}, m^{i}, \ldots, m^{i}, c^{*}, c^{*}, \ldots, c^{*}, a^{0 *}, a^{0 *}, \ldots, a^{0 *}\right)
$$

$m^{i}$, o minmax do jogador $i$, que, no nosso caso, é também a autarquia, como é fácil perceber, é jogado durante um total de $R$ períodos, e $a^{0 *}, S$ vezes num total de nS períodos.

Com relação à trajetória (33), podemos perceber que o jogador $i$ não obteria nenhuma vantagem ao tentar desviar-se de $m^{i}$. Além disso, nenhum jogador tem incentivos para desviar-se da trajetória estabelecida para os $\mathrm{nS}$ períodos da terceira fase ou fase de Nash. Nossa tarefa agora é escolher $S, R$ e $Q$, tal que ocorra o seguinte: a) nenhum jogador $j$ pode desviar-se da trajetória estabelecida para a segunda fase (ou fase de recompensa) se for ameaçado com a seguinte punição: os demais jogadores jogarão o equilíbrio de $G\left(T_{j}\right)$ que produz $z_{j}$ o maior número de vezes possível e então passarão a jogar o equilibrio de Nash que produz $w_{j}(1)$ durante o resto de $G(Q+R+n S) ; \mathrm{b})$ os jogadores $j \neq i$ não se podem beneficiar de um abandono da trajetória estabelecida para a fase de punição (primeira fase); c) o jogador $i$ obtém uma utilidade média menor do que $x_{i}+\omega$, onde $x_{i}=\left(\lambda v_{i}+(1-\lambda) z_{i}\right)$.

Começamos por escolher $S_{j}$ tal que

$$
P_{j}\left(c^{*} / b_{j}\left(c^{*}\right)\right)+n S_{j} w_{j}(1)<P_{j}\left(c^{*}\right)+S_{j} P_{j}\left(a^{0 *}\right),
$$

onde a notação $\left(c^{*} / b_{j}\left(c^{*}\right)\right)$ significa o resultado em que o j-ésimo elemento de $c^{*}$ é substituído por $b_{j}\left(c^{*}\right)$. Isto evita desvios durante a fase de recompensa.

Escolhemos então $S=\max \left(S_{1}, \ldots, S_{n}\right)$ e, a partir desta escolha, procuramos $Q$ e $R$ que satisfaçam simultaneamente, para $j \neq i$, as inequaçōes

$$
\begin{aligned}
& P_{j}\left(m^{i} / b_{j}\left(m^{i}\right)\right)+\left(Q+R+n S-T_{j}\right) z_{j}+\left(T_{j}-1\right) w_{j}(1)< \\
& <Q y_{j}^{i}+R\left(z_{j}+r\right)+S P_{j}\left(a^{0 *}\right),
\end{aligned}
$$

pois $y_{j}^{i}<z_{j}$, e

$$
Q v_{i}+R z_{i}+S P_{i}\left(a^{0 *}\right)<\left(x_{i}+\omega\right)(Q+R+n S)
$$


A desigualdade (35) é suficiente para evitar qualquer desvio em qualquer período da fase de punição. Isto ocorre porque a expressão à esquerda da desigualdade representa a utilidade máxima que o jogador $j$ poderia obter caso se desviasse de $m^{i}$ no período 1 e fosse em seguida punido pela repetição da trajetória correspondente ao equilíbrio de $G\left(T_{j}\right)$ o maior número de vezes possível, seguindo-se a repetição do pior equilíbrio de Nash para o jogador $j$ durante o restante do jogo. A expressão à direita, por sua vez, corresponde à utilidade que o jogador $j$ obteria cașo não se desviasse. 'Já (36) garante que a utilidade média de $i$ é menor do que $x_{i}+\omega$.

Reescrevendo (35) como

$$
\begin{aligned}
& Q\left(y_{j}^{i}-z_{j}\right)+R r>P_{j}\left(m^{i} / b_{j}\left(m^{i}\right)\right)+T_{j} z_{j}-S\left(P_{j}\left(a^{0 *}\right)-n z_{j}\right)+ \\
& +\left(T_{j}-1\right) w_{j}(1)
\end{aligned}
$$

e (36) como

$$
Q\left(v_{i}-x_{i}-\omega\right)+R\left(z_{i}-x_{i}-\omega\right)<-S\left(P_{j}\left(a^{0 *}\right)-n\left(x_{i}+\omega\right)\right),
$$

podemos perceber que uma condição suficiente para que possamos encontrar $Q$ e $R$ satisfazendo (35) e (36) é:

$$
\left(v_{i}-x_{i}-\omega\right) /\left(z_{i}-x_{i}-\omega\right)<\left(y_{i^{\prime}}^{i}-z_{i^{\prime}}\right) / r
$$

ou, rearranjando termos,

$$
x_{i}+\omega>\lambda v_{i}+(1-\lambda) z_{i},
$$

onde $\lambda$ está definido no lema. Ora, esta condição necessariamente se verifica porque $\omega>0$. Para entender porque esta é uma condição suficiente, basta perceber que (40) nos garante que a linha no espaço $(Q, R)$ definida por (38) é mais inclinada do que a definida por (37). Portanto, existe um equilíbrio perfeito em subjogos de $G(Q+$ $R+n S)$, onde a utilidade média do jogador $i$ não excede $x_{i}+\omega$.

Uma vez provado este lema, pode-se provar o seguinte teorema, cuja demonstração não apresentaremos pelo fato de que encontra-se na literatura sobre o assunto. ${ }^{29}$

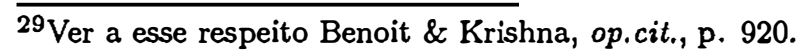


Teorema 2. Para $n=2$, se existe, para cada jogador $j$, um $a^{j 0}$ tal que $P_{j}\left(a^{j 0}\right)>w_{j}(1), j=1,2$, então toda utilidade $p$ individualmente racional e factivel pode ser obtido no limite a partir de um equilibrio perfeito em subjogos.

Este resultado nos indica que dois países, agindo não-cooperativamente e tendo como instrumento de ação suas políticas tarifárias, poderiam atingir utilidades médias superiores ao do equilibrio de Nash, ou seja, da guerra tarifária por ele representado, ou a qualquer utilidade que não estivesse sobre a fronteira eficiente de Pareto, desde que seguissem as estratégias analisadas na demonstração acima, ou seja, de punição em três fases. Supondo que o livre comércio seja preferível por ambos os países ao equilíbrio de Nash, poderíamos aproximar-nos, no limite, do utilidade a ele associada.

Voltando agora ao jogo de quotas, como ele não apresenta ao menos dois equilíbrios de Nash estáticos, e como este pré-requisito é essencial para demonstração do lema e, consequentemente, do teorema apresentados, fica evidente que não é possível provar a existência de outros equilíbrios perfeitos em subjogos que não a mera repetição do equilibrio de Nash estático, no caso, a autarquia.

Sendo assim, dada uma tarifa explícita, se impusermos uma quota igual ao volume de importações que prevaleceria com aquela tarifa, obteremos uma tarifa implícita igual à explícita. Entretanto, se tentarmos estipular uma estratégia baseada em quotas que, para cada valor da tarifa explícita estipulado na estratégia do jogo de tarifas, associa uma quota segundo esta orientação, não conseguiremos encontrar um equilíbrio perfeito em subjogos. Ou seja, não haverá ameaças críveis que impeçam desvios por parte dos países de trajetórias pré-estabelecidas.

Da mesma forma, se equivalência significar que, para cada tarifa explícita, podemos encontrar uma quota que produza o mesmo nível de utilidade, a extensão do jogo simples para o repetido é problemática. $^{30}$ Podemos imaginar uma estratégia baseada em quotas correspondente àquela baseada em tarifas, ou seja, a cada tarifa explícita associamos uma quota que produz o mesmo nível de utilidade. Entretanto, não passamos do terreno das estratégias. A

${ }^{30}$ Este conceito é devido a Shibata(1968). 
trajetória de resultados que surgirá no caso de quotas será diferente da do jogo de tarifas e não corresponderá a um equilíbrio perfeito em subjogos, a não ser que se refira à repetição do equilíbrio de Nash estático.

Portanto, podemos concluir pela não-equivalência entre tarifas e quotas num contexto de jogos finitamente repetidos com utilidades médias.

\section{Conclusão.}

Precisamos inicialmente enfatizar que o resultado de não-equivalência entre tarifas e quotas no contexto de jogos finitamente repetidos foi derivado sob a suposição de que os governantes não descontam os utilidades futuras quando da tomada de decisōes, ou seja, conferem o mesmo peso ao presente e ao futuro, o que não é muito razoável. Entretanto, como foi frisado anteriormente, decidimos utilizar utilidades médias na análise para podermos obter resultados mais "robustos".

Feita esta ressalva, podemos verificar que esse resultado tem várias implicações de ordem prática. $\mathrm{Na}$ verdade, ele significa que é possível, num ambiente não-cooperativo, haver um consenso entre os países que atuam no comércio mundial, com relação à adoção de políticas tarifárias mais liberais, que conduzam a utilidades maiores do que as obtidas com guerras tarifárias. O mesmo já não é possível quando o instrumento de política comercial usado são as quotas. Dessa forma, pcdemos redimensionar o papel do GATT no que diz respeito ao controle destes dois tipos de restrição ao comércio mundial.

A atuação do GATT no campo das tarifas pode dar-se a nível de regulamentação de ameaças críveis. Isto quer dizer que o GATT pode ter a função de dar uma conformação institucional a ameaças que, de outra forma, poderiam ser frustradas por consideraçōes geopolíticas, administrativas ou de outra natureza. Retornando ao campo teórico, isto significaria que a ameaça de um dos países de, por exemplo, punir um desvio de outro país através da chamada punição em três fases seria percebida pelos outros países como uma ameaça efetiva, ou seja, que seria implementada. Caso não houvesse a determinação do GATT, este tipo de ameaça poderia padecer da influência de fatores 
geopolíticos, por exemplo, como a importância de determinado país para a segurança de certa região geográfica.

$\mathrm{Na}$ área de quotas, o GATT poderia patrocinar acordos sobre flexibilizações das políticas comerciais dos países-membros, envolvendo, inclusive, outras formas de barreiras ao comércio, já que o ambiente não-cooperativo não seria propício para a obtenção de resultados positivos na liberalização do comércio.

Resta enfatizar que uma extensão óbvia deste trabalho seria para o caso de mais de dois bens e de dois países. Deve ter ficado claro que isto, entretanto, envolve complicaçōes consideráveis pelo fato de que a satisfação das condições do lema apresentado torna-se bem mais difícil de provar.

(Submetido em janeiro de 1992. Revisado em agosto de 1992)

\section{Referências}

Benoit, J. P. \& Krishna, V. 1985. "Finitely repeated games." Econometrica 53:905-922.

Bhagwati, J. 1965. "On the equivalence of tariffs and quotas." In Baldwin, R.E. and others, eds., Trade, Growth and the Balance of Payments: Essays in Honor of Gottfried Haberler. Chicago: Rand McNally.

Dixit, A. 1987. "Strategic aspects of trade policy." In Bewley, T. Advances in Economic Theory. Cambridge: Cambridge University Press.

Friedman, J.W. 1986. Game Theory with Applications to Economics. New York: Oxford University Press.

Fuedenberg \& Tirole. 1991. Game Theory. Cambridge: The MIT Press.

Jonhson, H. G. 1953/54. "Optimum tariffs and retaliation." Review of Economic Studies 21:142-153.

McMillan, J. 1986. Game Theory in International Economics. New York: Harwood Academic Publishers.

Rodriguez, C. A. 1974. "The non-equivalence of tariffs and quotas under retaliation." Journal of International Economics 4: 295-298. 
Shibata, H. 1968. "A note on the equivalence of tariffs and quotas." American Economic Review 58: 137-142.

Tower, E. 1975. "The optimum quota and retaliation." Review of Economic Studies 42:623-630.

Werlang, S. R. da Costa. 1988. "Estratégias de negociação em organizações internacionais: o ponto de vista da teoria dos jogos." Revista Brasileira de Economia 42.

Woodland, A.D. 1982. International Trade and Resource Allocation. Amsterdam: North-Holland. 